\title{
Significant Arterial Dissection
}

National Cancer Institute

\section{Source}

National Cancer Institute. Significant Arterial Dissection. NCI Thesaurus. Code C99709.

A dissection that impairs flow. This includes Type C (a persisting contrast medium extravasation) in the presence of ischemia, Type $D$ (a spiral filling defect with delayed but complete distal flow), Type E (persistent filling defect with delayed antegrade flow) and Type $F$ (filling defect with impaired flow and total occlusion). 